\title{
Adaptive Surface Modeling Using a Quadtree of Quadratic Finite Elements
}

\author{
G. P. Nikishkov \\ University of Aizu, Aizu-Wakamatsu 965-8580, Japan \\ niki@u-aizu.ac.jp \\ http://www.u-aizu.ac.jp/ niki
}

\begin{abstract}
This article presents special quadrilateral quadratic refinement elements, which provide geometry and field continuity across Tjunctions where two elements are connected to one side of a larger quadrilateral. The main idea in element refinement is to place one or more nodes outside the element area and to modify element shape functions in order to maintain continuity at refinement edges. Special refinement elements allow one to adaptively refine a mesh in such a way that it fits a quadtree data structure. An algorithm of surface modeling starts with a coarse mesh of quadratic quadrilateral elements. Adaptive mesh refinement is done in an iterative manner. At each iteration, the finite element equation system is solved to provide nodal locations with minimization of global approximation error. Elements with excessive local errors are split into four new elements. The mesh refinement iteration process is terminated when no element splits occur. The created mesh of quadratic quadrilaterals can be used directly in finite element analysis.
\end{abstract}

\section{Introduction}

Surface modeling is important for many fields of activity ranged from industrial CAD to animation in computer art. This paper is focussed on surface modeling for engineering applications, which use finite element analysis. The basic objective of this type of surface modeling can be stated as follows: generate a surface mesh that approximates a surface with specified accuracy and that can be used for finite element analysis.

Specified accuracy of surface approximation can be achieved with the use of adaptive algorithms. Adaptive mesh optimization algorithms for surface approximation have been considered in [1,2,3]. Duchineau et al. [1] presented an adaptive algorithm for constructing triangular meshes with guaranteed error bounds. Parajola [2] constructed restricted quadtrees for storing blocks of triangles in quadtree leafs. Minimization of global approximation error was done by Grosso et al. [3] using solution of a variational problem. However, the authors of these publications employed triangular elements. While triangular elements can be used in the finite element analysis, quadrilateral elements are preferable because of their better properties for solution of variational problems [4]. It is 
known that quadrilaterals with quadratic interpolating functions are more efficient than linear quadrilateral elements. Generation of a topologically regular quadrilateral mesh by a mapping technique is relatively easy [5]. However, performing local mesh refinement for a quadrilateral mesh is considered a difficult task [6. For example, a subdivision scheme for quadrilateral meshes proposed by Kobbelt [7] produces regular meshes with some singular vertices.

A natural process of local refinement for quadrilateral meshes is subdivision of one quadrilateral element into four smaller quadrilaterals. However, so-called "T-junctions" where two elements are connected to one side of a larger element are created after such subdivisions. Such meshes cannot be employed in visualization and modeling because of geometrical and functional discontinuity across T-junctions. Forting and Tanguy [8] considered connection of two Lagrangian 9 -node elements to one side of the same type larger element. Displacement compatibility was enforced by introduction of additional constraints in the global finite element equation system. This provides convergence of the finite element solution but the finite element mesh remains geometrically incompatible. Sederberg et al. 9] dealt with T-junction meshes using a generalization of B-splines, which they call T-splines.

The main contribution of this article is an introduction of special quadrilateral refinement elements, which provide continuity across element T-junctions. In refinement elements, one or more midside nodes are placed outside the element area and element shape functions are modified in order to maintain continuity at refinement edges. Shape functions for refinement elements are derived in Section 2. In Section 3, a finite element procedure for surface modeling with global error minimization is presented. Section 4 describes an iterative algorithm for adaptive surface approximation, which produces a quadtree of quadratic quadrilaterals with guaranteed local approximation error. In Section 5, an example of surface modeling is given.

\section{Refinement Quadrilaterals}

An isoparametric quadratic quadrilateral element 4 with eight nodes is depicted in Fig. 1,a. Any field (including coordinates) specified at element nodes is interpolated inside the element with the use of shape functions $N_{i}$, which are defined in local coordinates $\xi, \eta(-1 \leq \xi, \eta \leq 1)$ :

$$
\begin{aligned}
& u(\xi, \eta)=\sum N_{i}(\xi, \eta) u_{i}, \\
& N_{i}=\frac{1}{2}\left(1-\xi^{2}\right)\left(1+\eta \eta_{i}\right), \quad i=2,6, \\
& N_{i}=\frac{1}{2}\left(1+\xi \xi_{i}\right)\left(1-\eta^{2}\right), i=4,8, \\
& N_{i}=\frac{1}{4}\left(1+\xi \xi_{i}\right)\left(1+\eta \eta_{i}\right)-\frac{1}{2}\left(N_{i-1}+N_{i+1}\right) i=1,3,5,7,
\end{aligned}
$$

where $u(\xi, \eta)$ is a field value at some point inside the element; $u_{i}$ are field values at element nodes; $\xi_{i}$ and $\eta_{i}$ are values of the local coordinates $\xi$ and $\eta$ at the node $i$. 


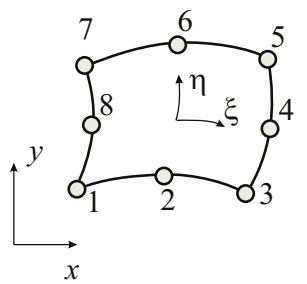

$a$

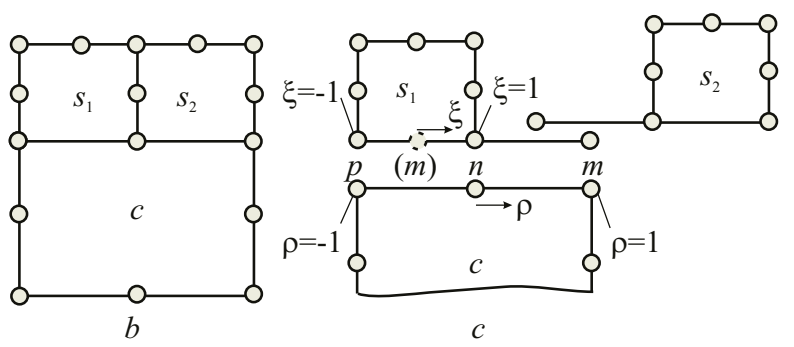

Fig. 1. a) Quadratic isoparametric quadrilateral element; b) Mesh refinement 1:2; c) Configuration of special elements

Using ordinary quadrilateral elements it is difficult to make mesh refinement. We present here special refinement elements that allow T-junctions as shown in Fig. 1.b. In special refinement elements, some midside nodes are moved outside the element area in order to provide geometry and field continuity. An idea of mesh refinement with special elements is illustrated in Fig. 1,c. Mesh T-junction is created using two refinement elements $s_{1}$ and $s_{2}$, which have nodes located outside of the element area.

Shape functions in the refinement element should provide continuity across Tjunction. In order to have continuity along the element edge $p-n-m$ (Fig. 11. c) the field value at point $\xi=0$ in the special element $s_{1}$ should be equal to the field value at point $\rho=-0.5$ in the conventional neighboring element $c$ : $u(\xi=0)=u(\rho=-0.5)$. Using interpolation (1), a compatibility equation can be written as:

$$
u(\xi=0)=u(\rho=-0.5)=\frac{3}{8} u_{p}+\frac{3}{4} u_{n}-\frac{1}{8} u_{m} .
$$

It is possible to demonstrate that the compatibility equation can be satisfied if the shape functions $N_{i}$ in the refinement element are modified as follows:

$$
N_{p}^{\prime}=N_{p}+\frac{3}{8} N_{m}, \quad N_{m}^{\prime}=-\frac{1}{8} N_{m}, \quad N_{n}^{\prime}=N_{n}+\frac{3}{4} N_{m} .
$$

Interpolation of a field, which is equal to 1 just at one corner node of the conventional element, is shown in Fig. 2,a. Same field interpolation in the refinement element is depicted in Fig. 2,b. Refinement elements preserve quadratic interpolation for both geometry and field modeling.
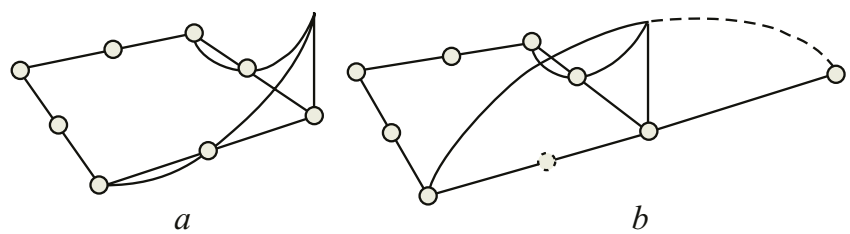

Fig. 2. Corner shape function in an ordinary quadratic quadrilateral (a) and in a refinement element 

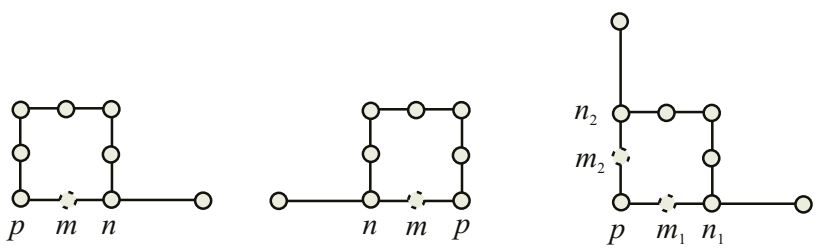

Fig. 3. Typical configurations of special refinement elements

Configurations of special refinement elements useful for quadtree mesh subdivision are shown in Fig. 3. If a special element contains two outside nodes as shown in Fig. 3] on the right, then modification (3) is applied twice using nodes $m_{1}$ and $m_{2}$ :

$$
\begin{aligned}
& N_{p}^{\prime}=N_{p}+\frac{3}{8}\left(N_{m_{1}}+N_{m_{2}}\right), \quad N_{m_{1}}^{\prime}=-\frac{1}{8} N_{m_{1}}, \quad N_{n_{1}}^{\prime}=N_{n_{1}}+\frac{3}{4} N_{m_{1}}, \\
& N_{m_{2}}^{\prime}=-\frac{1}{8} N_{m_{2}}, \quad N_{n_{2}}^{\prime}=N_{n_{2}}+\frac{3}{4} N_{m_{2}} .
\end{aligned}
$$

\section{Global Error Minimization}

Global error minimization of surface approximation can be done with the use of the variational approach to functional minimization. It is assumed that a surface that should be approximated is defined explicitly or by a point cloud. An element approximation error $E_{e}$ for a surface segment covered by element $e$ can be defined as:

$$
E_{e}=\int_{A_{e}}(f-u)^{2} d A=\int_{A_{e}}\left(f-\sum N_{i} u_{i}\right)^{2} d A,
$$

where $f$ is the specified surface height; $u$ is the height approximated by the eth element; $u_{i}$ are nodal values of $u ; N_{i}$ are the shape functions and $A_{e}$ is the element area. Element error can serve as a local error indicator for mesh refinement. Global approximation error is the sum of element errors:

$$
E=\int_{A}(f-u)^{2} d A=\sum_{e} E_{e}=\sum_{e} \int_{A_{e}}\left(f-\sum N_{i} u_{i}\right)^{2} d A .
$$

Minimization of the global error functional $E$ results in the following global finite element equation system:

$$
\mathbf{K U}=\mathbf{R}, \quad k_{i j}^{e}=\int_{A_{e}} N_{i} N_{j} d A, \quad r_{i}^{e}=\int_{A_{e}} N_{i} f d A,
$$

where $\mathbf{K}$ is a global "stiffness" matrix; $\mathbf{U}$ is a vector of nodal height values and $\mathbf{R}$ is a right-hand side vector. The global matrix $\mathbf{K}$ and the right-hand side vector $\mathbf{R}$ are composed of element "stiffness" matrices $\mathbf{k}_{e}$ and element vectors $\mathbf{r}_{e}$ using element connectivity information. 
Iterative methods can be efficiently used for solution of the finite element equation system (7) since good initial approximation for the solution vector $\mathbf{U}$ is available in the beginning of each refinement step.

\section{Surface Modeling Algorithm}

Surface modeling starts with a coarse mesh consisting of usual quadrilaterals. If the approximation domain is a quadrilateral area then it is possible to employ just one quadratic quadrilateral element in the beginning. According to the adaptive mesh refinement procedure, refinements are carried out for elements where an error indicator (5) is greater than a specified error tolerance.

The quadrilateral element that should be refined, is replaced by four quadrilaterals. Some or all of these new quadrilaterals are special refinement elements. Examples of one element refinement for the case of regular element surrounding and for the case of a partially refined mesh are shown in Fig. [
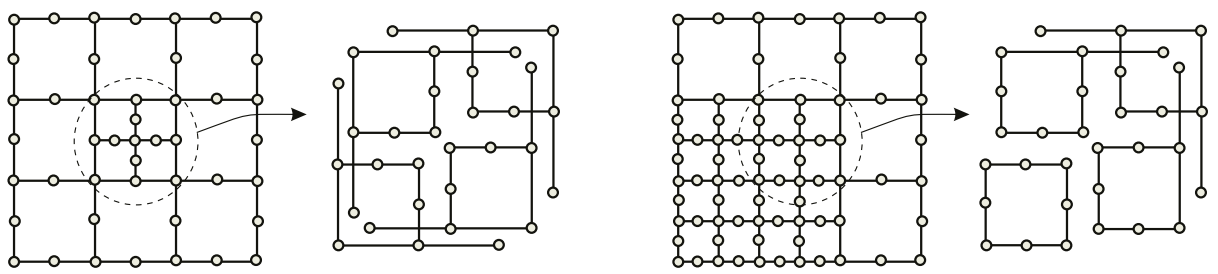

Fig. 4. Examples of 1:4 element refinement for the case of regular element surrounding and for the case of a partially refined mesh

Mesh data after any number of 1:4 element refinements is stored in a quadtree data structure. If to adopt that a quadtree root can be depicted as a square then each quadtree node corresponds to a smaller square. Internal nodes have exactly four children. Leafs (nodes without children) represent actual elements, which can be usual elements or special refinement elements. After splitting elements with excessive errors, the refined mesh can contain places where one element have a connection to more than two elements. This makes impossible to provide continuity across element boundaries. A quadtree where large squares can be adjacent to more than two smaller squares at any edge is called unbalanced [10]. Quadtree balancing is performed by additional element subdivisions. For any balanced quadtree, the geometry and field continuity can be ensured by using refinement elements with outside nodes.

An adaptive algorithm of surface modeling is organized as an iterative procedure. During each iteration, first, the finite element equation system (7) is assembled and solved. The height surface values are obtained for all mesh nodes. Then local element errors $E_{e}$ (5) are estimated and 1:4 splits of elements with excessive errors are performed. The element quadtree is balanced at the end of each iteration. Mesh refinement process is terminated if no splits occur during 
current iteration. Pseudo code of the surface modeling algorithm is presented below.

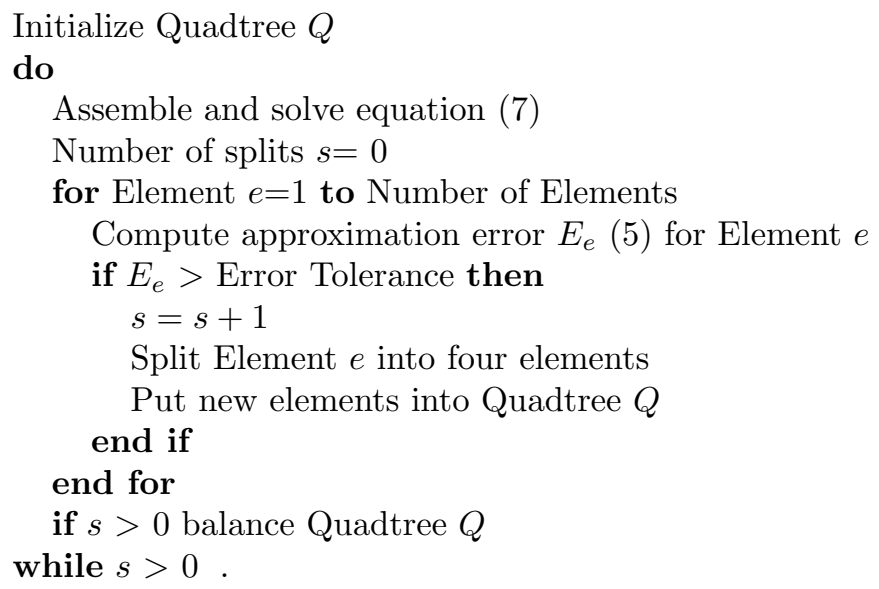

The algorithm of surface modeling has been implemented in Java. Surface is visualized using Java 3D.

\section{Example}

The proposed algorithm of adaptive surface modeling with a quadtree of quadratic quadrilaterals is demonstrated on the approximation of the following surface defined on a square domain:

$$
f=0.5 e^{-0.16\left(x^{2}+y^{2}\right)} \sin (2 x) \cos (2 y), \quad-5 \leq x \leq 5, \quad-5 \leq y \leq 5 .
$$

The height range for the above height function is $[-0.5,0.5]$ and the size in height direction is 1 . The following error measure is used for mesh refinement:

$$
\bar{E}_{e}=\sqrt{\frac{1}{A_{e}} \int_{A_{e}}(f-u)^{2} d A},
$$

where $f$ is the specified surface height; $u$ is the height approximation and $A_{e}$ is the element area. This error measure $\bar{E}_{e}$ is the modified element error (5): $E_{e}$ is divided by the element area and the square root is taken. The error indicator $\bar{E}_{e}$ is measured in length units and can be treated as some averaged absolute error over an element.

Results of surface approximation by quadtrees of quadratic quadrilateral elements are presented in Figures 5 and 6. Fig. 5 shows element quadtrees for error tolerance values 0.0005 (6 iterations) and 0.0001 ( 7 iterations). Visualization of the approximated surface (error tolerance 0.0005) with the use of Java 3D is presented in Fig. 6. 


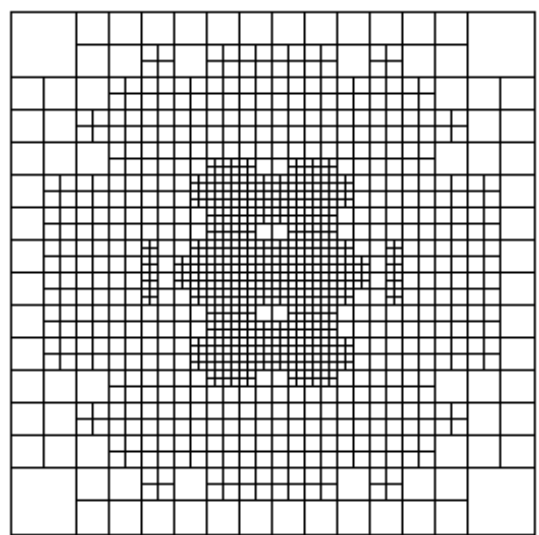

Error $=0.0005$

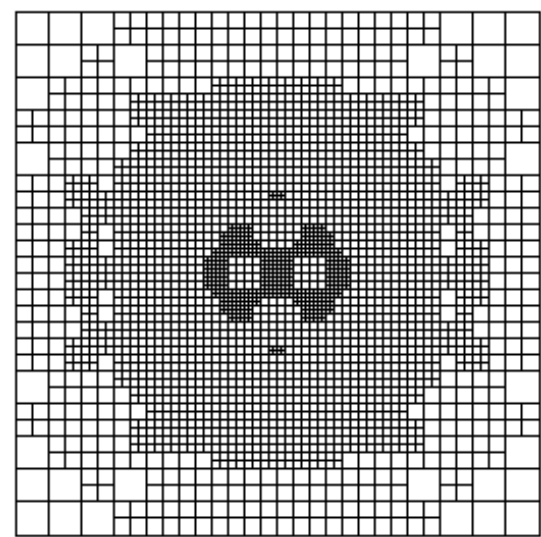

Error $=0.0001$

Fig. 5. Quadtrees of quadratic quadrilaterals characterized by different height approximation errors

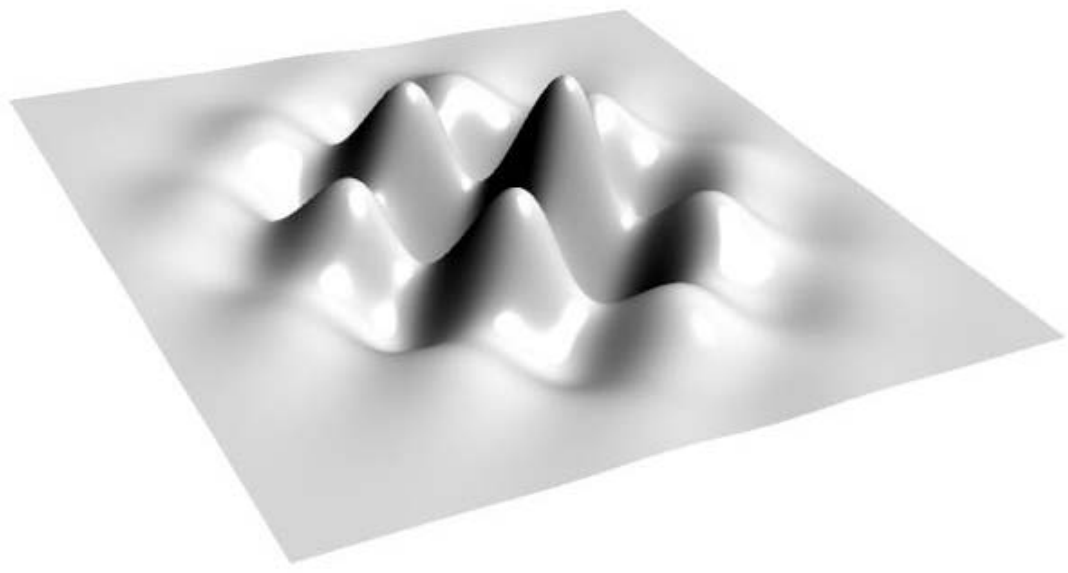

Fig. 6. Visualization of a surface approximated by quadratic quadrilaterals

\section{Conclusion and Future Work}

We have introduced special quadratic quadrilateral elements for adaptive surface modeling. Two special quadrilateral elements can be connected to one ordinary edge of a quadrilateral quadratic element. The special refinement elements are created by placement of one or two midside nodes outside the element area and by modification of element shape functions. The modified shape functions maintain geometry and field continuity across element T-junctions. It is worth noting that the refinement elements do not differ from standard quadratic finite elements and can be incorporated into existing finite element codes. 
Ordinary and special quadratic quadrilateral elements are used for surface approximation. Global approximation error is minimized by solution of a variational problem using the finite element method. A local element error indicator is utilized for adaptive mesh refinement. Elements with excessive local errors are subdivided into four elements each. At any refinement stage the element mesh is topologically equivalent to a quadtree. The quadtree data structure is used to store element data and to navigate through the mesh. Quadtree balancing is performed after each mesh refinement step in order to provide conforming connections of special and ordinary elements.

The proposed algorithm of surface modeling with a quadtree of quadratic quadrilaterals is demonstrated on the surface height approximation for a square domain. A surface mesh produced by the algorithm can be directly used in finite element analysis, where quadrilateral elements are considered more suitable than triangles.

Quadrilateral refinement elements can be employed for general surface modeling and for problems of the "surface on surface" type. For general surface modeling, a coarse starting mesh of quadrilateral elements topologically consistent with the modeled surface is created. Quadtree subdivisions are performed inside each element of the starting mesh. Mesh balancing should include balancing of quadtrees inside each starting element and balancing elements on the interelement boundaries of the starting mesh. Future research will follow this direction.

\section{References}

1. Duchineau, M. et al.: ROAMing terrain: real-time optimally adapting meshes. Procs of the IEEE Visualization 97 (1997) 81-88.

2. Pajarola, R.: Large scale terrain visualization using the restricted quadtree triangulation. Procs of the IEEE Visualization 98 (1998) 19-24.

3. Grosso, R., Lurig, C., Ertl, T.: The multilevel finite element method for adaptive mesh optimization and vizualization of volume data. Procs of the IEEE Visualization 97 (1997) 387-395.

4. Bathe, K.-J.: Finite Element Procedures. Prentice-Hall (1996).

5. Frey, P.J., George, P.-L.: Mesh Generation. Application to Finite Elements. Hermes (2000).

6. Zorin, D., Schröder, P.: Subdivision for modeling and animation. SIGGRAPH'00 Course Notes (2000).

7. Kobbelt, L.P.: A subdivision scheme for smooth interpolation of quad-mesh data. Procs of EUROGRAPHICS'98, Tutorial (1998).

8. Fortin, M., Tanguy, P.: A non-standard mesh refinement procedure through node labelling. Int. J. Numer. Meth. Eng. 20 (1984) 1361-1365.

9. Sederberg, T.W., Zheng, J., Bakenov, A., Nasri, A.: T-splines and T-NURCCs. ACM Trans. on Graphics, 22 (2003) 477-484.

10. de Berg, M., van Kreveld, M., Overmars, M. Schwarzkopf, O.: Computational Geometry. Algorithms and Applications. Springer (2000). 